\title{
Sirtuins: The Future Insight
}

\section{Suvarna BS}

\author{
Dept of Pharmacology, \\ Melaka Manipal Medical College
}

\section{Corresponding Author}

Beena S Suvarna

Ex-Professor of Pharmacology

Melaka Manipal Medical College, Manipal.

Email: drbsusheel@yahoo.co.uk

Citation

Suvarna BS. Sirtuins: The Future Insight. Kathmandu Univ Med J 2012;38(2):77-82.

\begin{abstract}
Sirtuins are evolutionary conserved NAD+ dependent acetyl-lysine deacetylases and ADP ribosyltransferases dual-function enzymes involved in the regulation of metabolism and lifespan. Sirtuins represent a promising new class of III NAD dependent histone deacetylases that regulate a number of physiological processes, originally identified in yeast. Sirtuins regulate various normal and abnormal cellular and metabolic processes, including tumorgenesis, neurodegeneration and processes associated with type 2 diabetes and obesity. Several age-related diseases such as Alzheimer's disease and longevity have also been linked to the functions of sirtuins. Because of these associations, the identification of small molecules sirtuin modulators has been of significant interest.
\end{abstract}

\section{KEY WORDS}

Sirtuins, SirT1-7, histone deacetylases, epigenetics.

\section{INTRODUCTION}

Sirtuins are a class III NAD dependent histone deacetylases that regulate a number of physiological processes, play an important role in the regulation of metabolism, aging, oncogenesis and cancer progression. ${ }^{1}$ Histone deacetylases (HDACs) are a part of a group of proteins that regulate acetylation. These enzymes are responsible for removing the acetyl group from $\varepsilon$-lysine residues and consequently are involved in gene repression and heterochromatin formation. This role also makes HDACs key players in epigenetic regulation and management of specific spatialtemporal programs of expression, such as development and cellular differentiation. ${ }^{2}$

Sirtuins are also implicated in determining the balance between apoptosis, cell survival and cell proliferation. ${ }^{1}$ In Humans, seven sirtuins isoforms have been identified. ${ }^{3}$ The three mammalian sirtuins (SIRT1, SIRT6, SIRT7) are localised to the nucleus, SIRT2 localised to the cytoplasm and SIRT4, SIRT3, SIRT5 localised to mitochondria. SIRT1 is most extensively studied, has more than a dozen known substrates and is a guardian against cellular oxidative stress and DNA damage. Moreover, SIRT1 plays a prominent role in metabolic tissues, such as pancreas, fat and liver. SIRT6 and SIRT7 may be important regulators of DNA damage and metabolism respectively. ${ }^{3}$ The sirtuin family of histone deacetylases (HDACs) was named after their homology to the Saccharomyces cerevisiae gene silent information regulator 2 (Sir2). ${ }^{4}$

Epigenetic modulations (transformations) play an important role in regulating gene expression, life span and tumorgenesis. They are not modulated (regulated) by inhibitors of class I, II and IV HDACs. The deacetylase activity of sirtuins is controlled by the cellular (NAD)/(NADH) ratio, where NAD+ works as an activator, while nicotinamide and $\mathrm{NADH}$ act as inhibitors. Expressed from bacteria to humans, sirtuins seem to preferentially target non-histone proteins, although little is currently known about target specificity and selectivity. ${ }^{5}$ Two reactions may be catalysed by sirtuins: deacetylation and ADP-ribosylation. In both reactions, the cleavage of NAD+ is the initial step. Sirtuins (SirT1, SirT2, SirT3, SirT5 and SirT7) catalyse a deacetylation reaction on lysine residues of target proteins using NAD+ as cofactor and releasing nicotinamide with the production of 2-O-acetyl-ADP ribose. In contrast, SirT4 and SirT6 catalyse an ADP-ribosylation reaction, in which ADP-ribosyl moiety is transferred to the substrate. ${ }^{6}$ SirT1, the closest to yeast Sir2 in terms of sequence, is a proto-member of the family. 
SIRT1 is most extensively studied, has more than a dozen known substrates, and is a guardian against cellular oxidative stress and DNA damage. Moreover, SIRT1 plays a prominent role in metabolic tissues, such as pancreas, fat, and liver. ${ }^{7}$

SirT1 mediates heterochromatin formation through deacetylation of $\mathrm{K} 26$ on histone $\mathrm{H} 1, \mathrm{~K} 9$ on histone $\mathrm{H} 3$ and $\mathrm{K} 16$ on histone $\mathrm{H} 4$. SirT1 is also involved in the deacetylation of non-histone proteins, which can be divided into three groups: (1) transcription factors ( $p 53$, FOXO, E2F1, BCL6, $\mathrm{p} 53, \mathrm{Rb})(2)$ DNA repair proteins (3) signalling factors. Due to its ability to deacetylate a variety of substrates, SirT1 is involved in a broad range of physiological functions, including control of gene expression, metabolism, tumorgenesis and aging. ${ }^{3}$

Human SirT2 co-localises with microtubules in cytoplasm. Thus, knockdown of SirT2 results in tubulin hyperacetylation. SirT2 also bind FOXO3a, reducing its acetylation levels. ${ }^{7,8}$ Mammalian SIRT2 is a predominantly cytoplasmic protein co-localizes with tubulin, and can deacetylate a number of substrates in vitro, including a-tubulin and histones, although the physiological consequences of a-tubulin deacetylation by SIRT2 are not yet clear. ${ }^{9-11}$ Cell culture studies demonstrate SIRT2 may be important in regulating mammalian cell cycle. SIRT2 protein levels increase during mitotic phase of the cell cycle and its over expression delays mitosis. ${ }^{9}$ Consistent with the idea that SIRT2 may restrain the cell cycle, expression of this sirtuin is down-regulated in human gliomas, compared with normal brain samples. ${ }^{12}$

SIRT3 was the first sirtuin shown to be localized to the mitochondria of mammalian cells. ${ }^{11,13,14}$ SIRT3 is localized to the mitochondrial matrix and cleavage of its signal sequence is necessary for enzymatic activity. ${ }^{14}$ SIRT3 deacetylates multiple substrates in vitro including histone peptides and tubulin. ${ }^{10,13,14}$ SIRT3 is expressed in brown adipose tissue and induced by cold exposure. ${ }^{15}$ Moreover, the deacetylase activity of SIRT3 is reported to be required for the induction of uncoupling protein 1 (UCP-1). SIRT3 also appears to regulate mitochondrial functions, as its over-expression increases respiration, while decreasing reactive oxygen species production. ${ }^{15}$

Two recent studies demonstrate that SIRT3 may regulate the activity of acetyl-CoA synthetase (AceCS), representing a striking, conserved activity with the bacterial sirtuin, cobB. ${ }^{14,16-18}$ AceCS uses acetate, CoA, and ATP to form acetylCoA, which is an intermediate in the TCA cycle, and is also required for cholesterol and fatty acid synthesis. Acetylation of mitochondrial AceCS (AceCS2) inactivates the enzyme, whereas deacetylation by SIRT3 activates it. Interestingly, SIRT1 can deacetylate and activate the cytosolic form of AceCS (AceCS1). These data suggest that SIRT3 may play a role in regulating the entry of carbons from acetate into central metabolism. In sum, SIRT3 may be especially important under conditions of energy limitation-i.e., during fasting or CR to ensure full incorporation of dietary or ketone-derived acetate into metabolism.

SIRT4 is another mitochondrial protein that regulates energy usage. SIRT4 lacks detectable deacetylase activity,but demonstrates ADP-ribosyltransferases activity. ${ }^{10,11}$ SIRT4 plays an important role in regulating amino acid-stimulated insulin secretion (AASIS) in pancreatic ß-cells by ADPribosylating and inhibiting glutamate dehydrogenase (GDH). ${ }^{19} \mathrm{GDH}$ converts glutamate into $\alpha$-ketoglutarate, a TCA cycle intermediate. GDH-activating mutations cause hyperinsulinism in humans showing that this enzyme regulates insulin secretion by gating the flow of amino acids into central metabolism in ß-cells. ${ }^{20}$

The third mitochondrial sirtuin, SIRT5 localised in the intermembrane space, deacetylases carbamoyl phosphate synthetase 1 (CPS 1), an enzyme which is the first and rate-limiting step in urea cycle. Deacetylation of CPS 1 by SIRT5 results in activation of CPS 1 enzymatic activity. Animal study showed in SIRT5-deficient mice failed to upregulate CPS 1activity and showed hyper ammonia during fasting. Similar effects are also observed on high protein diet or calorie restriction. These data indicate SIRT5 as an emerging role in the metabolic adaptation to fasting, high protein diet and calorie restriction. ${ }^{21}$

The mitochondrial localization of SIRT3-5 is especially intriguing because mitochondrial dysfunction is associated with mammalian aging and many diseases, including diabetes, neurodegenerative diseases, and cancer. ${ }^{22}$ Lifespan analysis of animals with varying SIRT3-5 level has not been performed; however, there is growing evidence linking mitochondrial sirtuins with regulating energy usage and even human lifespan.

The last two sirtuins, SirT6 and SirT7 have recently been characterized. SirT6 exerts an NAD+ -dependent histone H3K9 deacetylase action able to modulate telomeric chromatin functions. ${ }^{23}$ SirT6 has a role in genome stability. In mammalian cells subjected to oxidative stress, SirT6 is recruited on DNA double-strand breaks (DSB) and stimulates repair through both homologous and nonhomologous end-joining recombination.

SirT7 is a predominantly nucleolar protein associated with active rRNA genes (rDNA), where it interacts with RNA polymerase I and histones. ${ }^{3}$ SirT7 does not seem to have any NAD+ dependent activity. ${ }^{3}$ Animal studies showed that SIRT7 prevents apoptosis in response to stress response in the heart. ${ }^{24}$ Interestingly, SIRT7 expression correlates with growth and it is abundant in tissues with high proliferation, such as liver, spleen, and testes. ${ }^{25}$ By contrast, SIRT7 expression is absent or low in non-proliferating tissues, like heart, brain, and muscle. Recent work has shown that SIRT7 may regulate cellular growth and metabolism. ${ }^{25}$ In the nucleolus, SIRT7 associates with rDNA and interacts with RNA polymerase I (Pol I). Overexpressing SIRT7 increases rRNA transcription and RNA inhibition of SIRT7 decreases transcription, showing that this sirtuin activates Pol I transcription. ${ }^{25}$ 
Some sirtuins may relocalise depending on cell or tissue type, development stage, stress condition and metabolic status suggesting that their localization is important for regulating function. ${ }^{26}$

Sirtuins and Diseases:

Diabetes Mellitus: SIRT1 down regulates p53 activity, increasing lifespan, cell survival and neuroprotection; it also deacetylates peroxisome proliferator - activated receptor gamma and its co-activator 1 alpha, promoting fat mobilization, increasing mitochondrial size and number, and positively regulating insulin secretion. Resveratrol might help in the treatment or prevention of obesity and in preventing the aging related decline in heart function and neuronal loss. New SIRT1 activators are 1000 times more effective than Resveratrol according to recent research. ${ }^{27}$

Antiageing: Ageing is characterized by a progressive deterioration functions and metabolic processes. In aging and in diseases associated with the elderly, such as Alzheimer's or Parkinson's, the loss of cells in vital structures or organs may be related to several factors, among which the production of reactive oxygen species (ROS) by mitochondria is a common denominator, one that leads to DNA damage, apoptosis and death. It is well established that reducing food intake (caloric restriction) extends the life span in a wide range of species. The protein implicated in this protective process is the silent information regulator 2 (SIRT2, SIRT1 in mammals), an enzyme that belongs to a nicotinamide adenine dinucleotide (NAD) + dependent protein deacetylases. The link between the role of sirtuins, caloric restriction and longevity was first described in S. Cerevisiae. Lifespan extension has not been observed in yeast lacking Sir $2 .{ }^{28}$ SIRs regulate gene silencing, DNA repair, rDNA recombination and ageing, apart from regulating programmed cell death. In this context, increasing SIRT1 has been found to protect cells against amyloid beta-induced ROS production and DNA damage, thereby reducing apoptotic death in vitro. The aging process is associated with telomere erosion. Recent studies have demonstrated that reduction or removal of SirT6 results in telomere dysfunction and end-to-end chromosomal fusions. Symptoms displayed in the absence of SirT6 are similar to those caused by a premature aging disease known as Werner's syndrome. Little known about other sirtuins, but no evidence suggests their involvement with telomere function, formation and stability. ${ }^{29}$ SirT1 has been shown to promote replicative senescence in response to chronic oxidative stress by regulation of the p19ARF senescence regulator, which in turn positively regulates p53 via inhibition of MDM2, a protein mediating p53 degradation. ${ }^{30}$

It has also been demonstrated that Alzheimer's and Huntington's disease neurons are rescued by the overexpression of SIRT1, induced by either caloric restriction or administration of Resveratrol, a potential activator of this enzyme. The therapeutic use of Resveratrol and other related compounds utilise SIRT1 pathway modulators in treating aging-related brain disorders. ${ }^{31}$

In models of Huntington's disease, SirT1 activation reduces cell death by inhibition of NFKB signalling. Alzheimer's disease is also related to sirtuin action given that SirT1 over expression in the brain of mice reduces $\beta$-Amyloid production and the formation of plaques. The $\beta$-Amyloid peptide generates protein aggregates (plaques) in the brain of patients. ${ }^{32}$ Recent studies also suggest that SirT1 may benefit learning and memory by activating the gene for brain derived neurotrophic factor also protects against amyotrophic lateral sclerosis. ${ }^{33,34}$

Cancer: Interest in sirtuins has grown in the last decade, mainly because of their critical role in different biological processes, such as regulation of gene expression, control of metabolic processes, apoptosis and cell survival, DNA repair, development, neuroprotection and inflammation. SIRT1 associates with the tumour suppressor protein p536 and regulates protein levels of $\mathrm{p} 53$ through deacetylation of residue L382, which destabilises p53, thereby promoting cell survival. ${ }^{6,35-38}$ Over-expression of SIRT1 has been found in cancer cells to promote tumour cell survival. ${ }^{39}$ SIRT1 can deacetylase the DNA repair factor Ku70 which sequesters the pro-apoptotic protein $B A X$ in the mitochondria thus preventing apoptosis. ${ }^{40}$

Nevertheless, SirT1 seems to play contradictory roles, both as tumour suppressor or tumour promoter. ${ }^{41,42}$ The initial evidence that SirT1 acts as tumour promoter derives from its repressive effect on tumour suppressor p53. ${ }^{41}$ By interacting physically with p53, SirT1 blocks its functions through deacetylation at the C-terminal K382 residue. ${ }^{43}$ SirT1 over expression in cancer represses p53- dependent cell-cycle arrest and apoptosis in response to DNA damage and oxidative stress. DNA damage-induced acetylation of p53 leads to its activation. In response to damage, SirT1 binds to and deacetylates p53, thus reducing its functional and transcriptional activities. Over expression of SirT1 disrupts p53-dependent pathways, resulting in a significant reduction in the cell's ability to respond to stress and DNA damage. The inhibition of SirT1 potentiates p53-dependent apoptosis. ${ }^{41,44}$

Decreased SirT1 levels have been reported for glioma, bladder, prostate and ovarian cancers. Some studies have suggested that SirT1 over expression in APC-/+ mice reduces rather than increases colon cancer formation. ${ }^{45}$ This action seems to be caused by SirT1 deacetylation of $\beta$-catenin, which promotes cytoplasmic localization of the nuclear localized oncogenic form of $\beta$-catenin. SirT1 deficiency also causes reduced DNA double strand break repair and radiation sensitivity. ${ }^{46} \mathrm{~A}$ potential tumour suppressor role has also been proposed for the other human sirtuins.26 This hypothesis is supported by several findings such as the reduction of SirT2 in a large number of human brain tumour cell lines, and its involvement in cellcycle progression. SirT3 is the only mitochondrial sirtuin 
implicated in tumorgenesis. Its reduction in several cancers leads to an increase in ROS (reactive oxygen species) production which results in enhanced tumour growth. ${ }^{47}$ SirT5 over expression may be found in a study of pancreatic cancer. ${ }^{48}$

Recently, a role for SirT6 and SirT7 in tumorgenesis has been proposed. SirT6 might be involved as a result of its control of the NFKB pathway and DNA double strand repair. SirT7, whose expression inversely correlates with the tumorigenic potential in several murine cell lines, displays increased expression levels in breast cancer. ${ }^{49,50}$

Gene expression and deacetylase activity of the class III histone deacetylase SIRT1 are up-regulated in cancer cells due to oncogene over expression or loss of function of tumour suppressor genes. SIRT1 induces histone deacetylation and methylation, promoter CpG island methylation, transcriptional repression and deacetylation of tumour suppressor proteins. SIRT1 may play a critical role in tumour initiation, progression and drug resistance by blocking senescence and apoptosis and promoting cell growth and angiogenesis. SIRT1 inhibitors have shown promising anticancer effects in animal models of cancer. ${ }^{51}$

Sirtuins is emerging as a promising antitumor strategy whereby Salermide, a reserve amide with a strong in vitro inhibitory effect on Sirt1 and Sirt2 has been studied. Salermide was well tolerated by mice at concentrations up to $100 u M$ and promoted tumour - specific cell death in a wide range of human cancer cell lines. The studies showed the apoptotic effect of Salermide to the reactivation of proapoptotic genes epigenetically repressed in cancer cells by SIRT1. ${ }^{52}$

\section{Sirtuin modulators:}

A great deal of research interest has been focused on the identification of small chemical compounds that modulate these proteins. Many inhibitors of sirtuins have been proposed for therapy against neurodegenerative diseases and cancer. ${ }^{3}$ Sirtinol is mainly an inhibitor of the yeast Sir2 and human SirT2. ${ }^{53}$ Its inhibitory effect on growth has been described in human breast, lung and prostate cancer cells. Another potent and selective inhibitor of SirT2 is AGK2. It was demonstrated that the inhibition of SirT2 protects against dopaminergic cell death in a Drosophilia model of Parkinson's disease. ${ }^{33}$ Suramin is a potent inhibitor of many sirtuins. However its nephrotoxicity limits its therapeutic use. $^{54,55}$ Cambinol and Salermide are inhibitors of SirT1 and SirT2. ${ }^{56,57}$ Cambinol shows the potent activity against Burkitt lymphoma cell lines by a mechanism involving BCL6 acetylation. Salermide induces apoptosis in cancer cells in a p53 independent manner. The indole derivative, EX-527 is a cell permeable selective inhibitor of SirT1. Treatment with EX-527 increases acetylation at K382 of p53 after DNA damage induction in primary human mammary epithelial cells and several cell lines. ${ }^{58}$ The direct interaction between
SirT1 and p53 highlights potential SirT1 involvement in p53 functions including differentiation, DNA repair and aging. Currently EX-527 is in phase 1 clinical trials for the treatment of Huntington's disease. In preclinical studies this inhibitor was shown to reduce neuronal death. Tenovins, a family of small molecule inhibitors have a potential therapeutic interest for the development of new drugs that act against tumour cells over expressing SirT1. ${ }^{59}$ Uv15008 has recently been reported as a novel epigenetic modifier able to inhibit simultaneously histone deacetylases, sirtuins and DNA methyltransferases. This compound induces cancer cell sensitive death in several human models. This action is independent of p53; $\mathrm{Bcl}$-2-modifying factor (BMF) and TNF-related apoptosis-inducing ligand (TRAIL), affecting the growth of tumour cells deficient or mutated for these factors. This finding may potentiate its application in therapy by overcoming potential drug resistance limits. ${ }^{60}$

\section{CONCLUSION}

Over the decade, the study of sirtuins has made remarkable progress and expanded our knowledge. Sirtuins influences several cellular processes. Growing evidence has underlined their involvement in many diseases. Although SirT1 has been extensively researched, a better understanding of its involvement in pathogenesis is required. However, our understanding of sirtuin biology is still far from complete and many questions remain to be answered. The development of potent sirtuin modulators may revert the disease process and possibly extend healthy human lifespan. Emerging from research on the sirtuins is a growing appreciation that they are a very complicated biological response system that influences many other regulator molecules and pathways in complex manners. Recently, growing interest in sirtuin modulation has led to discovery and characterization of small molecules able to modify sirtuin activity. While it may be years before we know whether sirtuins regulate mammalian lifespan, current data suggests that these proteins are regulated by diet and in turn, regulate multiple facets of physiology, making them interesting therapeutic targets for metabolic and neurodegenerative diseases. Thus, to achieve the goal of a therapeutic intervention of aging, it will be important to fully elucidate the functions of all seven sirtuins and in many different tissues.

\section{REFERENCES}

1. Balcerczyk A, Pirols L. Therapeutic potential of activators and inhibitors of sirtuins. Biofactors. 2010 ; 36 (5):383-93.

2. Ahringer J. NuRD and SIN3 histone deacetylase complexes in development. Trends Genet. 2000; 16 (8): 351-56.

3. Alcain FJ, Villalba JM. Sirtuin inhibitors. Expert Opin Ther Pat .2009; 19(3): 283-94.

4. Dali-Youcef N, Lagouge M, Froelich S. Sirtuins: the magnificent seven, 
function, metabolism and longevity. Ann Med. 2007; 39(5):335-45.

5. Vaquero $A$. The conserved role of sirtuins in chromatin regulation. Int. J. Dev. Biol. 2009; 53:303-22.

6. Yamamoto $\mathrm{H}$, Schoonjans $\mathrm{K}$, Auwerx J. Sirtuin function in heath and disease. Mol. Endocrinol. 2007; 21(8):1745-55.

7. Marcia C, Leonard P, Guarente1. Mammalian sirtuins-emerging roles in physiology, aging, and calorie restriction. Genes \& Dev. 2006;20: 2913-21

8. Vassilopoulos A, Fritz KS, Dennis R. Petersen DR. The human sirtuin family: Evolutionary divergences and functions. Hum Genomics. 2011; 5(5): 485-96.

9. Dryden S.C., Nahhas F.A., Nowak J.E. Role for human SIRT2 NADdependent deacetylase activity in control of mitotic exit in the cell cycle. Mol. Cell. Biol .2003; 23(9) :3173-85.

10. North B.J., Marshall BL., Borra M.T. The human Sir2 ortholog, SIRT2, is an NAD+-dependent tubulin deacetylase. Mol. Cell. 2003; 11(2):437444.

11. Michishita E., Park J.Y., Burneskis J.M. Evolutionarily conserved and nonconserved cellular localizations and functions of human SIRT proteins. Mol. Biol. Cell. 2005; 16 (10):4623-35

12. Hiratsuka M., Inoue T., Toda T. Proteomics-based identification of differentially expressed genes in human gliomas: Down-regulation of SIRT2 gene. Biochem Biophys Res Commun. 2003; 309 (3):558-66.

13. Onyango P., Celic I., McCaffery J.M. SIRT3, a human SIR2 homologue, is an NAD-dependent deacetylase localized to mitochondria. Proc. Natl. Acad. Sci. 2002; 99(21): 13653-58.

14. Schwer B., Bunkenborg J., Verdin R.O. Reversible lysine acetylation controls the activity of the mitochondrial enzyme acetyl-CoA synthetase 2. Proc Natl Acad Sci. 2006; 103(27): 10224-29.

15. Shi T., Wang F., Stieren E., Tong, Q. SIRT3, a mitochondrial sirtuin deacetylase, regulates mitochondrial function and thermogenesis in brown adipocytes. J Biol Chem. 2005; 280(14): 13560-567.

16. Hallows W.C., Lee S., Denu J.M. Sirtuins deacetylate and activate mammalian acetyl-CoA synthetases. Proc Natl Acad Sci. 2006; 103(27): 10230-35.

17. Tsang A.W., Escalante-Semerena J.C. CobB, a new member of the SIR2 family of eucaryotic regulatory proteins, is required to compensate for the lack of nicotinate mononucleotide:5,6-dimethylbenzimidazole phosphoribosyltransferase activity in cobT mutants during cobalamin biosynthesis in Salmonella typhimurium LT2. J Biol Chem. 1998; 273(48):31788-94

18. Starai V.J., Celic I., Cole R.N. Sir2-dependent activation of acetyl-CoA synthetase by deacetylation of active lysine. Science.2002;298(5602):2390-92.

19. Haigis M.C., Mostoslavsky R., Haigis K.M. SIRT4 inhibits glutamate dehydrogenase and opposes the effects of calorie restriction in pancreatic ß cells. Cell. 2006; 126 (5):941-54. http://www.ncbi.nlm. nih.gov/pubmed/16959573

20. Stanley C.A., Lieu Y.K., Hsu B.Y. Hyperinsulinism and hyperammonemia in infants with regulatory mutations of the glutamate dehydrogenase gene. N. Engl. J. Med. 1998; 338(19):1352-57.

21. Nakagawa T, Guarente L. Urea cycle regulation by mitochondrial sirtuin, SIRT5. Aging. 2009;1(6): 578-81

22. Wallace, D.C. A mitochondrial paradigm of metabolic and degenerative diseases, aging, and cancer: A dawn for evolutionary medicine. Annu Rev Genet.2005; 39:359-407. http://www.ncbi.nlm.nih.gov/pmc/ articles/PMC2821041/.

23. Michishita E, McCord RA, Berber E. SIRT6 is a histone H3 lysine 9 deacetylase that modulates telomeric chromatin. Nature. 2008 ;Mar 27: 452(7186):492-6
24. Vakhrusheva O, Smolka C, Gajawada P. Sirt7 increases stress resistance of cardiomyocytes and prevents apoptosis and inflammatory cardiomyopathy in mice. Circ Res. 2008;102 (6): 703-10.

25. Ford E., Voit R., Liszt G.Mammalian Sir2 homolog SIRT7 is an activator of RNA polymerase I transcription. Genes Dev. 2006; 20 (9):10751080.

26. McGuinness D, McGuinness DH, McCaul JA et al. Sirtuins, bioageing and cancer. J. Aging Res.2011; 235754 (Pubmed)

27. Jiang WJ. Sirtuins: novel targets for metabolic disease in drug development. Biochem Biophys Res Commun. 2008 Aug 29; 373(3):341-4.

28. Lin SJ, Defossez PA, Guarente L. Requirement of NAD and Sir2 for life -span extension by calorie restriction in Saccharomyces cerevisiae. Science. 2009; 289: 2126-28.

29. Westphal CH, Dipp MA, Guarente L. A therapeutic role for sirtuins in diseases of aging? Trends Biochem Sci. 2007; 32 (12): 555-60.

30. Chua KF, Mostoslavsky R, Lombard DB. Mammalian SIRT1 limits replicative life span in response to chronic genotoxic stress. Cell Metab. 2005; 2 (1):67-68.

31. Merce Pallas, Esther Verdaguer, Marta Tajes. Recent patents on CNS Drug Discovery. Bentham Science Publishers. Vol3, no.1, 2008. Pp.61$69 \mathrm{http}: / /$ www.benthamscience.com/contents-JCode-PRN-Vol00000003-Iss-00000001.htm.

32. Outiero TF, Marqes $\mathrm{O}$, Kazantev A. Therapeutic role of sirtuins in neurodegenerative disease. Biochem Biophys Acta. 2008; 363-69.

33. Gao J, Wang WY, Mao YW . A novel pathway regulates memory and plasticity via SIRT1 and miR-134. Nature 2010; 466 (7310):1105-09.

34. Dohoon Kim, Minh Dang Nguyen, Matthew M Dobbin. SIRT1 deacetylase protects against neurodegeneration in models for Alzheimer's disease and amyotrophic lateral sclerosis. EMBO J. 2007 July 11; 26(13): 3169-3179. http://www.ncbi.nlm.nih.gov/pmc/ articles/PMC1914106/.

35. Bouras T, Fu MF, Sauve AA. SIRT1 deacetylation and repression of $p 300$ involves lysine residues 1020/1024 within the cell cycle regulatory domain 1. Journal of Biological Chemistry. 2005; 280:16456-60.

36. Haigas MC \& Guarente LP. Mammalian sirtuins-emerging roles in physiology,aging and calorie restriction. Genes and Development. 2006; 20:2913-21.

37. Rodgers JT, Lerin C, Haas W. Nutrient control of glucose homeostasis through a complex of PGC-1 alpha and SIRT1. Nature 2005; 434:11318.

38. Zhao WH, Kruse JP, Tang $Y$. Negative regulation of the deacetylase SIRT1 by DBC1. Nature 2008; 451:587-90.

39. Ota H, Tokunaga E, Chang K. Sirt1 inhibitor, Sirtinol, induces senescence-like growth arrest with attenuated Ras-MAPK signalling in human cancer cells. Oncogene. 2006b; 25:176-185.

40. Cohen HY, Lavu S, Bitterman KJ. Acetylation of the $\mathrm{C}$ terminus of Ku70 by CBP and PCAF controls Bax-mediated apoptosis. Molecular Cell 2004a; 13:627-38.

41. Deng CX. SirT1, is it a tumour promoter or tumour suppressor? Int J Biol Sci. 2009; 5(2): 147-152.

42. Bosch PL, Vaquero A. The dual role of sirtuins in cancer. Genes Cancer.2011;2(6): 648-62.

43. Vaziri H, Dessain SK, Eaton E . hSIR2 (SIRT1) functions as an NADdependent p53 deacteylase. Cell. 2001; 107(2): 149-59.

44. Rahman S, Islam R. Mammalian Sirt1: insight on its biological functions. Cell Commun. Signal. 2011; 9: 11.

45. Firestein R, Blander G, Michan S. The sirt1 deacetylase suppress intestinal tumorigenesis and colon cancer growth. PLoS ONE. 2008. http://www.plosone.org/article/info:doi\%2F10.1371\%2Fjournal. 


\section{pone. 0002020}

46. Wang RH, Sengupta K, Li C, Kim HS. Impaired DNA damage response, genome instability and tumorigenesis in SIRT1 mutant mice. Cancer cell. 2008;14(4): 312-23.

47. Kim HS, Patel K, Muldoon JK. SIRT3 is a mitochondria-localised tumor suppressor required for maintenance of mitochondrial integrity and metabolism during stress. Cancer Cell. 2010; 17(1): 41-52.

48. Ouaissi M, Sielezneff I, Silvestre R. High histone deacetylase 7 (HDAC7) expression is significantly associated is significantly associated with adenocarcinomas of the pancreas. Ann Surg Oncol. 2008; 15(8):231828.

49. Vakhrusheva O, Braeuer D, Liu Z. Sirt7 dependent inhibiton of cell growth and proliferation might be instrumental to mediate tissue integrity during aging. J Physiol Pharmacol. 2008; 59 (suppl 9): 20112.

50. Ashraf N, Zino S, Macintrye A. Altered sirtuin expression is associated with node-positive breast cancer. Br J Cancer 2006; 95(8): 1056-61.

51. Liu T, Lui PY, Marshall GM. The critical role of the class III histone deacetylase SIRT1 in cancer. Cancer Res. 2009; 69(5): 1702-05.

52. Lara E, Mai A, Calvanese V. Salermide, a Sirtuin inhibitor with a strong cancer-specific proapoptotic effect. Oncogene. 2009; 28 (6): 781-91.

53. Grozinger CM, Chao ED, Blackwell HE . Identification of a class of small molecule inhibitors of the sirtuin family of NAD - dependent deacetylase $s$ by phenotypic screening. J Biol Chem. 2001; 276(42):
38837-43.

54. Schuetz A, Min J, Antoshenko T. Structural basis of inhibition of the human NAD+ dependent deacetyalse SIRT5 by suramin. Structure. 2007;15(3): 377-89.

55. Trapp J, Meier R, Hongwiset D. Structure-activity studies on suramin analogues as inhibitors of NAD + dependent histone deacetylases (sirtuins). Chem Med Chem. 2007; 2(10): 1419-31.

56. Heltweg B, gatbonton T, Schuler A. Antitumor activity of a smallmolecule inhibitor of human silent information regulator 2 enzymes. Cancer Res. 2006; 66 (8): 4368-77.

57. Lara S, Hollick JJ, Calvanese V . Salermide, a sirtuin inhibitor with a strong cancer-specific proapoptotic effect. Oncogene. 2009; 28(6):781-91.

58. Solomon JM, Pasupuleti R, Xu L. Inhibition of SIRT1 catalytic activity increases $\mathrm{p} 53$ acetylation but does not alter cell survivl following DNA damage. Mol Cell Biol Vol .2006; 26(1): 28-38.

59. Lain S, Hollick JJ, Campbell J. Discovery in vivo activity and mechansim of action of a small molecule p53 activator. Cancer Cell. 2008; 13(5): 454-63.

60. Nebbioso A, Pereira R, Khanwalkar H. Death receptor pathway activator and increase of ROS production by the triple epigenetic inhibitor, UV15008. Mol Cancer ther. 2011; 10(12): 2394-404. 